\title{
Delay Dependent Robust Exponential Stability Criterion for Perturbed and Uncertain Neutral Systems with Time Varying Delays
}

\author{
Issam Amri, Dhaou Soudani, Mohamed Benrejeb \\ Unit research LA.R.A., Ecole Nationale d'Ingénieurs de Tunis, ENIT, BP 37, 1002 Tunis Tunisia. \\ amri_issam@yahoo.fr,dhaou.soudani@enit.rnu.tn,mohamed.benrejeb@enit.rnu.tn
}

\begin{abstract}
This paper deals with the issue of robust exponential stability of uncertain neutral system with time varying delay and nonlinear perturbations. Using Lyapunov-Krasovskii functional, new sufficient delay dependent stability conditions have been derived in terms of Linear Matrix Inequalities LMIs solved using efficient convex optimization algorithms. Neither model transformation, nor estimating techniques for cross terms, nor free weighting matrices are involved in this work. Numerical examples are considered to show the efficiency of the proposed stability approach.
\end{abstract}

Keywords: Uncertain neutral systems; Time varying delays; Nonlinear perturbations; Exponential stability; Delay dependent stability; Linear matrix inequality.

\section{Introduction}

The phenomena of time delays are frequently encountered in engineering dynamic systems, for instance in chemical process, in network control systems, in long transmission and so on. It is well known that the existence of time delays in a system may be a major source of instability, oscillations and poor performance. In view of this, considerable attention has been devoted to the problem of stability and robustness of time delays systems for several decades, see for example [1-27], and the references therein. The developed stability results can be classified into two types: delay dependent stability results, which are concerned with the size of the delay and usually give the maximum delay bounds for making the system stable, and the delay independent stability results, which can be applied with arbitrary delay's size. Generally, delay dependent stability conditions are less conservative than delay independent ones, especially when the time delays are small.

In general, dynamical time delays systems models can be described as two types of functional differential equations. The first one concerns the retarded type which contains delays only in its states, whereas the second concerns the neutral system, being a special case of time delay system, which involved time delay in both state and state derivative simultaneously. Such system can be found in such places as population ecology [18], distributed networks containing lossless transmission lines [17], heat exchangers, robots in contact with rigid environments, etc. In recent years, stability issue in various neutral time delay systems have been widely investigated in many reports $[8,11,13,14$, $16,26]$. Moreover, the stability for the systems with time varying delays will be an important focus that described systems more physical than the constant time delays cases $[1,3,4,6,8,14,19,20,27]$. It is well known that in practice, systems almost present some uncertainties and nonlinear perturbations. Thus, many methods have been proposed to deal with uncertainties and nonlinear perturbations in the literature, and much attention has been paid on robust stability analysis by using the Lyapunov-Krasovskii functional approach $[1,4,6,8,11,14,15,19$ $22,26]$.

In [21], a parameterized neutral model transformation was utilized. Based on a model transformation technique, [6] presents delay dependent stability criteria by using a Lyapunov-Krasovskii functional approach. In [13], stability conditions for uncertain systems with time varying delay and nonlinear perturbations was developed by applying a descriptor model transformation and a decomposition technique of the delay term matrix. The estimation approaches for bounding the cross terms show in [14] may bring some conservatism. Furthermore, free weighting matrices have been employed in a lot of papers, such as [9], because they can increase the freedoms to search the Lyapunov matrices and reduce the conservatisms. 
On another hand, the problem of exponential stability has also been considered by some researchers $[1-4,7-9,11,15,16,20,23]$ because it is also important indices to get the convergence rates of prescribed time delay systems.

In these contexts, the issue of exponential stability for uncertain neutral systems with time varying delays and nonlinear perturbations remains open, which motivates [8] and this paper but by adding norm-bounded uncertainties to system under consideration.

The purpose of this paper is to investigate the problems of delay dependent robustly exponential stability for uncertain neutral system with time varying delays and nonlinear perturbations. We present a new sufficient condition based on a combination of Lyapunov-Krasovskii functional and Linear Matrix Inequalities LMIs which can be efficiently solved by standard convex optimization algorithms [5]. Neither model transformation, nor estimating techniques for cross terms, nor free weighting matrices are involved in this work. Some numerical examples are included to show the effectiveness of our approach and to illustrate the applicability of the developed results.

\section{Notations}

$$
\begin{array}{ll}
A^{T} & : \text { Transpose of matrix } A \\
S>0 & : S \text { symmetric positive definite } \\
& \text { matrix } \\
\lambda_{\text {Max }}(A) & : \text { Maximum (minimum) } \\
\left(\lambda_{\min }(A)\right) & \begin{array}{l}
\text { eigenvalue of a symmetric } \\
\text { matrix } A
\end{array} \\
\|\cdot\| & : \text { Euclidean vector norm } \\
I & : \text { Identity matrix } \\
* & : \text { Symmetric terms in a } \\
& \text { symmetric matrix }
\end{array}
$$

\section{System Description and Preliminaries}

Consider the following neutral system with time varying delays and nonlinear perturbations [6]:

$$
\begin{aligned}
\dot{x}(t) & =A x(t)+A_{h} x(t-h(t))+C \dot{x}(t-h(t)) \\
& +f(t, x(t))+g(t, x(t-h(t))) \\
x(t) & =\phi(t), \quad \dot{x}(t)=\varphi(t), \forall t \in[-h, 0]
\end{aligned}
$$

where $x(t) \in \mathfrak{R}^{n}$ is the state vector, and $A$, $A_{h}, C$, are known real constant matrices with appropriate dimensions, the time delay, $h(t)$, is a time varying continuous function that satisfies:

$0 \leq h(t) \leq h, \quad \dot{h}(t) \leq d$

$\phi(),. \varphi($.$) are the initial condition functions$ that are continuously differentiable on $[-h, 0]$. The time varying nonlinear functions $f(t, x(t))$ and $g(t, x(t-h(t)))$ are unknown and represent the nonlinear perturbations with respect to the current state $x(t)$ and delayed state $x(t-h(t))$. These functions satisfy $f(t, 0)=0, g(t, 0)=0$, and:

$f^{T}(t, x(t)) f(t, x(t)) \leq \gamma^{2} x^{T}(t) x(t)$

$g^{T}(t, x(t-h(t))) g(t, x(t-h(t))) \leq \beta^{2} x^{T}(t-h(t)) x(t-h(t))(4)$

where $\gamma$ and $\beta$ are positive scalars. For simplicity, we note $f:=f(t, x(t))$ and $g:=g(t, x(t-h(t)))$.

The following lemmas and definition will be essential for the development of our results.

\section{Definition 1 [8]}

The system (1) is exponentially stable, if there exist positive constants $\alpha$ and $\delta, \delta \geq 1$ such that for all $x(t)$, the following inequality holds:

$$
\|x(t)\| \leq \delta e^{-\alpha t}\|\psi\|_{h}, \forall t \geq 0
$$

where $\alpha$ is the decay rate and

$$
\|\psi\|_{h}=\sup _{-h \leq \theta \leq 0} \sqrt{\|\varphi(\theta)\|^{2}+\|\phi(\theta)\|^{2}} \text {. }
$$

\section{Lemma 1}

Consider the function:

$$
V(t)=\int_{t-h(t)}^{t} \int_{s}^{t} f(\theta) d \theta d s
$$

The first derivative $\dot{V}(t)$ is given by:

$$
\dot{V}(t)=h(t) f(t)-(1-\dot{h}(t)) \int_{t-h(t)}^{t} f(s) d s
$$




\section{Lemma 2 [5]}

Let $M, N$ and $K$ be given $n \times n$ real matrices such that $K>0$ and $K=K^{T}$. Then:

$$
\left[\begin{array}{cc}
M & N \\
N^{T} & -K
\end{array}\right]<0 \Leftrightarrow M+N K^{-1} N^{T}<0
$$

\section{Lemma 3 [25]}

For any constant matrix $R=R^{T}>0$ and scalar $h>0$, such that the following integral is defined, then:

$h \int_{t-h}^{t} x^{T}(s) R x(s) d s \geq \int_{t-h}^{t} x^{T}(s) d s R \int_{t-h}^{t} x(s) d s$

\section{Lemma 4 [10]}

Let $\Pi, H, F$ and $G$ real matrices of appropriate dimensions such that $F^{T}(t) F(t) \leq I$, then for any scalar $\varepsilon>0$, the following inequality holds:

$\Pi+H F(t) G+G^{T} F^{T}(t) H^{T} \leq \Pi+\varepsilon^{-1} H H^{T}+\varepsilon G^{T} G(10)$

\section{Lemma 5 [25]}

Let $f(x), y_{1}(x), \ldots, y_{k}(x)$ be some nonnegative functional or functions, and define the following conditions:

(a) $f(x) \geq 0$

(b) $\tau_{1} \geq 0, \ldots, \tau_{k} \geq 0$, such that $f(x)-\sum_{j=1}^{k} \tau_{j} y_{j}(x) \geq 0$

Then (b) implies (a).

\section{Robust Exponential Stability for Neutral Time Varying Delay Systems with Nonlinear Perturbations}

Formulation of a practically computable criterion is proposed in this section to check the exponential stability of the nonlinear perturbed neutral system (1). The following theorem provides a robustly delay dependent exponential stability criterion in terms of LMIs optimization approaches.

\section{Theorem 1}

For given scalars $\alpha>0$ and $h>0$, the nonlinear neutral system described by (1) is robustly exponentially stable with a decay rate $\alpha$ if there exist positive definite symmetric $n \times n$ matrices $P, w_{1}, w_{3}, Q_{1}, Q_{3}$, $H_{1}, H_{2}$ and any $n \times n$ matrices $w_{2}, Q_{2}$ such that the following LMIs hold:

$$
\Sigma=\left[\begin{array}{cccccccc}
\Sigma_{11} & \Sigma_{12} & \Sigma_{13} & -e^{-20 h_{1}} w_{2}^{T} & \Sigma_{15} & \Sigma_{16} & h A^{T} w_{3} & A^{T} Q_{3} \\
* & \Sigma_{22} & \Sigma_{23} & e^{-20 h} w_{2}^{T} & \Sigma_{25} & \Sigma_{26} & h h_{h}^{T} w_{3} & A_{h}^{T} Q_{3} \\
* & * & \Sigma_{33} & 0 & \Sigma_{35} & \Sigma_{36} & h C^{T} w_{3} & C^{T} Q_{3} \\
* & * & * & -e^{-20 h} w_{1} & 0 & 0 & 0 & 0 \\
* & * & * & * & \Sigma_{55} & \Sigma_{56} & 0 & 0 \\
* & * & * & * & * & \Sigma_{66} & 0 & 0 \\
* & * & * & * & * & * & -w_{3} & 0 \\
* & * & * & * & * & * & * & -Q_{3}
\end{array}\right]<0(11)
$$

$\left[\begin{array}{cc}w_{1} & w_{2} \\ w_{2}^{T} & w_{3}\end{array}\right]>0,\left[\begin{array}{ll}Q_{1} & Q_{2} \\ Q_{2}^{T} & Q_{3}\end{array}\right]>0$

with:

$$
\begin{aligned}
\Sigma_{11} & =2 \alpha P+P A+A^{T} P+h^{2} w_{1}+h^{2} A^{T} w_{2}^{T}+h^{2} w_{2} A \\
& +Q_{2} A+A^{T} Q_{2}^{T}+Q_{1}-e^{-2 \alpha h} w_{3}+\gamma^{2} H_{1} \\
\Sigma_{12} & =P A_{h}+h^{2} w_{2} A_{h}+Q_{2} A_{h}+e^{-2 \alpha h} w_{3} \\
\Sigma_{13} & =P C+h^{2} w_{2} C+Q_{2} C \\
\Sigma_{15} & =\Sigma_{16}=P+Q_{2}+h^{2} w_{2}+h^{2} A^{T} w_{3}+A^{T} Q_{3} \\
\Sigma_{22}= & -(1-d) e^{-2 \alpha h} Q_{1}-e^{-2 \alpha h} w_{3}+\beta^{2} H_{2} \\
\Sigma_{23} & =-(1-d) e^{-2 \alpha h} Q_{2} \\
\Sigma_{25} & =\Sigma_{26}=h^{2} A_{h}^{T} w_{3}+A_{h}^{T} Q_{3} \\
\Sigma_{33} & =-(1-d) e^{-2 \alpha h} Q_{3} \\
\Sigma_{35} & =\Sigma_{36}=h^{2} C^{T} w_{3}+C^{T} Q_{3} \\
\Sigma_{55} & =h^{2} w_{3}+Q_{3}-H_{1} \\
\Sigma_{56} & =h^{2} w_{3}+Q_{3} \\
\Sigma_{66} & =h^{2} w_{3}+Q_{3}-H_{2}
\end{aligned}
$$

\section{Proof of Theorem 1}

Let consider the Lyapunov-Krasovskii functional in the following form:

$V(t, x(t))=V_{1}(t, x(t))+V_{2}(t, x(t))+V_{3}(t, x(t))(13)$ 
with:

$$
\begin{aligned}
& V_{1}(t, x(t))=e^{2 \alpha t} x^{T}(t) \operatorname{Px}(t) \\
& V_{2}(t, x(t))=h \int_{t-h}^{t} \int_{s}^{t} e^{2 \alpha s} \zeta^{T}(\theta) W \zeta(\theta) d \theta d s \\
& V_{3}(t, x(t))=\int_{t-h(t)}^{t} e^{2 \alpha \theta} \zeta^{T}(\theta) Q \zeta(\theta) d \theta \\
& \zeta^{T}(\theta)=\left[x^{T}(\theta) \dot{x}^{T}(\theta)\right], \\
& \text { and } P>0, W=\left[\begin{array}{ll}
w_{1} & w_{2} \\
w_{2}^{T} & w_{3}
\end{array}\right]>0, Q=\left[\begin{array}{ll}
Q & Q_{2} \\
Q_{2}^{T} & Q_{3}
\end{array}\right]>0
\end{aligned}
$$

The time derivative of $V_{1}$ leads to:

$$
\begin{aligned}
& \dot{V}_{1}(t, x(t))=e^{2 \alpha t}\left\{x^{T}(t)\left[2 \alpha P+P A+A^{T} P\right] x(t)\right. \\
& +x^{T}(t-h(t)) A_{h}^{T} P x(t)+\dot{x}^{T}(t-h(t)) C^{T} P x(t) \\
& +f^{T} P x(t)+g^{T} P x(t)+x^{T}(t) P A_{h} x(t-h(t)) \\
& \left.+x^{T}(t) P C \dot{x}(t-h(t))+x^{T}(t) P f+x^{T}(t) P g\right\}
\end{aligned}
$$

Taking the time derivative of $V_{2}$ gives that:

$$
\dot{V}_{2}(t, x(t))=h^{2} e^{2 \alpha t} \zeta^{T}(t) W \zeta(t)-h \int_{t-h}^{t} e^{2 \alpha s} \zeta^{T}(s) W \zeta(s) d s
$$

Obviously, for any scalar $s \in[t-h, t]$ :

$$
e^{2 \alpha(t-h)} \leq e^{2 \alpha s} \leq e^{2 \alpha t}
$$

So, it follows:

$$
\begin{gathered}
-h \int_{t-h}^{t} e^{2 \alpha s} \zeta^{T}(s) W \zeta(s) d s \leq-h \int_{t-h}^{t} e^{2 \alpha(t-h)} \zeta^{T}(s) W \zeta(s) d s \\
\leq-h(t) \int_{t-h(t)}^{t} e^{2 \alpha(t-h)} \zeta^{T}(s) W \zeta(s) d s \\
\dot{V}_{2}(t, x(t)) \leq e^{2 \alpha t}\left\{h^{2} \zeta^{T}(t) W \zeta(t)\right. \\
\left.-h(t) e^{-2 \alpha h} \int_{t-h(t)}^{t} \zeta^{T}(s) W \zeta(s) d s\right\}
\end{gathered}
$$

According to lemma 3 , the following inequality holds:

$$
\begin{aligned}
-h(t) e^{-2 \alpha h} \int_{t-h(t)}^{t} \zeta^{T}(s) W \zeta(s) d s \\
\leq-e^{-2 \alpha h} \int_{t-h(t)}^{t} \zeta^{T}(s) d s W \int_{t-h(t)}^{t} \zeta(s) d s
\end{aligned}
$$

Then, an upper bound for $\dot{V}_{2}$ is obtained as:

$$
\begin{gathered}
\dot{V}_{2}(t, x(t)) \leq e^{2 \alpha t}\left\{h^{2} \zeta^{T}(t) W \zeta(t)\right. \\
\left.-e^{-2 \alpha h} \int_{t-h(t)}^{t} \zeta^{T}(s) d s W \int_{t-h(t)}^{t} \zeta(s) d s\right\} \\
\leq e^{2 \alpha t}\left\{h^{2} x^{T}(t) w_{1} x(t)+2 h^{2} x^{T}(t) w_{2}\right. \\
{\left[A x(t)+A_{h} x(t-h(t))+C \dot{x}(t-h(t))+f+g\right]} \\
+h^{2}\left[A x(t)+A_{h} x(t-h(t))+C \dot{x}(t-h(t))+f+g\right]^{T} w_{3} \\
{\left[A x(t)+A_{h} x(t-h(t))+C \dot{x}(t-h(t))+f+g\right]} \\
-e^{-2 \alpha h}[x(t)-x(t-h(t))]^{T} w_{3}[x(t)-x(t-h(t))] \\
-e^{-2 \alpha h}[x(t)-x(t-h(t))]^{T} w_{2}^{T} \int_{t-h(t)}^{t} x(s) d s \\
-e^{-2 \alpha h} \int_{t-h(t)}^{t} x^{T}(s) d s w_{2}[x(t)-x(t-h(t))] \\
\left.-e^{-2 \alpha h} \int_{t-h(t)}^{t} x^{T}(s) d s w_{1}^{t} \int_{t-h(t)}^{t} x(s) d s\right\}
\end{gathered}
$$

The time derivatives of $V_{3}$ is:

$$
\begin{aligned}
\dot{V}_{3} & =e^{2 \alpha t}\left[\zeta^{T}(t) Q \zeta(t)-(1-\dot{h}(t)) e^{-2 \alpha h(t)} \zeta^{T}(t-h(t)) Q \zeta(t-h(t))\right] \\
& \leq e^{2 \alpha t}\left[\zeta^{T}(t) Q \zeta(t)-(1-d) e^{-2 \alpha h} \zeta^{T}(t-h(t)) Q \zeta(t-h(t))\right] \\
& \leq e^{2 \alpha t}\left[\zeta^{T}(t) Q \zeta(t)-(1-d) e^{-2 \alpha h} \zeta^{T}(t-h(t)) Q \zeta(t-h(t))\right] \\
& \leq e^{2 \alpha t}\left\{x^{T}(t) Q_{1} x(t)-2 x^{T}(t) Q_{2}\right. \\
& {\left[A x(t)+A_{h} x(t-h(t))+C \dot{x}(t-h(t))+f+g\right] } \\
& +\left[A x(t)+A_{h} x(t-h(t))+C \dot{x}(t-h(t))+f+g\right]^{T} \\
& Q_{3}\left[A x(t)+A_{h} x(t-h(t))+C \dot{x}(t-h(t))+f+g\right] \\
& -(1-d) e^{-2 \alpha h} x^{T}(t-h(t)) Q_{1} x(t-h(t)) \\
& -2(1-d) e^{-2 \alpha h} x^{T}(t-h(t)) Q_{2} \dot{x}(t-h(t)) \\
& \left.-(1-d) e^{-2 \alpha h} \dot{x}^{T}(t-h(t)) Q_{3} \dot{x}(t-h(t))\right\}
\end{aligned}
$$

From equations (3) and (4), since the following two inequalities hold:

$\gamma^{2} x^{T}(t) x(t)-f^{T} f \geq 0$

$\beta^{2} x^{T}(t-h(t)) x(t-h(t))-g^{T} g \geq 0$

Therefore, there exist positive definite matrices $R_{1}$ and $R_{2}$ satisfying the inequalities:

$$
\begin{aligned}
& \gamma^{2} x^{T}(t) R_{1} x(t)-f^{T} R_{1} f \geq 0 \\
& \beta^{2} x^{T}(t-h(t)) R_{2} x(t-h(t))-g^{T} R_{2} g \geq 0
\end{aligned}
$$


From equations (13-24), and based on the Sprocedure, given in lemma 5 , there exist nonnegative scalars $\tau_{1} \geq 0$ and $\tau_{2} \geq 0$ such that:

$$
\begin{aligned}
& \dot{V}(t, x(t)) \leq \dot{V}_{1}(t, x(t))+\dot{V}_{2}(t, x(t))+\dot{V}_{3}(t, x(t)) \\
& +e^{2 \alpha t} \gamma^{2} x^{T}(t) \tau_{1} R_{1} x(t)-e^{2 \alpha t} f^{T} \tau_{1} R_{1} f \\
& +e^{2 \alpha t} \beta^{2} x^{T}(t-h(t)) \tau_{2} R_{2} x(t-h(t))-e^{2 \alpha t} g^{T} \tau_{2} R_{2} g
\end{aligned}
$$

By setting $H_{i}=\tau_{i} R_{i} \geq 0$ for $i=1,2$, the time derivative $\dot{V}$ is upper bounded as:

$\dot{V}(t, x(t)) \leq e^{2 \alpha t} \mu^{T}(t) \Theta \mu(t)$

with:

$$
\mu^{T}(t)=\left[x^{T}(t) x^{T}(t-h(t)) \dot{x}^{T}(t-h(t)) \int_{t-h(t)}^{t} x^{T}(s) d s f^{T} g^{T}\right]
$$

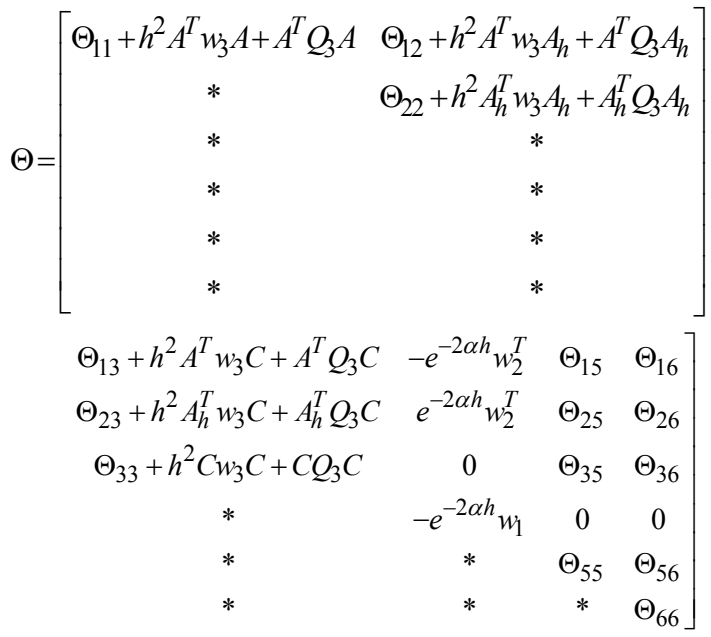

where $\Theta_{i j}=\Sigma_{i j}, i, j=1, \ldots, 6$, are the same as defined in the Theorem 1.

Using Shur's complement (lemma 2), $\Theta<0$ is equivalent to $\Sigma<0$, hence, that implies $\dot{V}(t, x(t))<0$ which ensures system (1) is robustly asymptotically stable for all nonlinear uncertainties (3) and (4) by Lyapunov-Krasovskii theory. This result leads to $V(t, x(t))<V(t, x(0))$. Then:

$$
\begin{aligned}
& V(t, x(0))=x^{T}(0) P x(0)+h \int_{-h}^{0} \int_{s}^{0} e^{2 \alpha s} \zeta^{T}(\theta) W \zeta(\theta) d \theta d s \\
& +\int_{-h(0)}^{0} e^{2 \alpha \theta} \zeta^{T}(\theta) Q \zeta(\theta) d \theta
\end{aligned}
$$

$$
\begin{aligned}
& \leq \lambda_{\operatorname{Max}}(P)\|\psi\|_{h}^{2}+h \lambda_{\operatorname{Max}}(W) \int_{-h}^{0} \int_{s}^{0} e^{2 \alpha s} \zeta^{T}(\theta) \zeta(\theta) d \theta d s \\
& +\lambda_{\operatorname{Max}}(Q) \int_{-h(0)}^{0} e^{2 \alpha \theta} \zeta^{T}(\theta) \zeta(\theta) d \theta \\
& \leq\left(\lambda_{\operatorname{Max}}(P)+h^{3} \lambda_{\operatorname{Max}}(W)+h \lambda_{\operatorname{Max}}(Q)\right)\|\psi\|_{h}^{2}
\end{aligned}
$$$$
\leq \sigma\|\psi\|_{h}^{2}
$$

with:

$$
\sigma=\lambda_{\operatorname{Max}}(P)+h^{3} \lambda_{\operatorname{Max}}(W)+h \lambda_{\operatorname{Max}}(Q) .
$$

Furthermore, one can see that:

$$
V(t, x(t)) \geq V_{1}(t, x(t))
$$

Then, the following inequality is obtained:

$$
V(t, x(t)) \geq e^{2 \alpha t} \lambda_{\min }(P)\|x(t)\|^{2}
$$

It follows from equations (25-27) that:

$$
e^{2 \alpha t} \lambda_{\min }(P)\|x(t)\|^{2} \leq V(t, x(t)) \leq \sigma\|\psi\|_{h}^{2}
$$

which leads to:

$$
\|x(t)\| \leq \sqrt{\frac{\sigma}{\lambda_{\min }(P)}} e^{-\alpha t}\|\psi\|_{h} \equiv \delta e^{-\alpha t}\|\psi\|_{h}
$$

with $\delta=\sqrt{\frac{\sigma}{\lambda_{\min }(P)}} \geq 1$. Thus, by definition 1 , the system (1) is robust exponentially stable with exponential convergence rate $\alpha$. This completes the proof of Theorem 1.

\section{Remark 1}

The proposed exponential stability conditions (11) and (12) are Linear Matrix Inequality LMIs. Hence, by iteratively solving the LMIs given in Theorem 1, with respect to $h$ for fixed $d, \alpha, \gamma, \beta$, it is easy to compute the maximum upper bound of the allowable delay $h$ guaranteeing the robustly exponential stability of the system (1) using efficient convex optimization algorithms [5].

\section{Remark 2}

For the case of $C=0$, which is studied in [6, $11,13,19,25,26]$, the system (1) can be rewritten as: 


$$
\begin{aligned}
& \dot{x}(t)=A x(t)+A_{h} x(t-h(t))+f(t, x(t))+g(t, x(t-h(t))) \\
& x(t)=\phi(t) \quad \forall t \in[-h, 0]
\end{aligned}
$$

Most of the existing delay dependent stability conditions require a constraint with the delay derivative $d<1$, and this constraint may cause conservativeness. Using similar method as in Theorem 1, we obtain a robust exponential stability criterion of the system (26) independently of $d$ that can be arbitrarily large. This result is shown in the following corollary.

\section{Corollary 1}

For given scalars $\alpha>0$ and $h>0$, the time delay system described by (30) is robustly exponentially stable with a decay rate $\alpha$ if there exist positive definite symmetric $n \times n$ matrices $P, w_{1}, w_{3}, Q_{1}, H_{1}, H_{2}$ and any $n \times n$ matrix $w_{2}$ such that the following LMIs hold:

$$
\Gamma=\left[\begin{array}{cccc}
\Gamma_{11} & \Gamma_{12} & -e^{-2 \alpha h} w_{2}^{T} & P+h^{2} w_{2}+h^{2} A^{T} w_{3} \\
* & \Gamma_{22} & e^{-2 \alpha h} w_{2}^{T} & h^{2} A_{h}^{T} w_{3} \\
* & * & -e^{-2 \alpha h} w_{1} & 0 \\
* & * & * & h^{2} w_{3}-H_{1} \\
* & * & * & * \\
* & * & * & * \\
& & P+h^{2} w_{2}+h^{2} A^{T} w_{3} & h A^{T} w_{3} \\
& & h^{2} A_{h}^{T} w_{3} & h A_{h}^{T} w_{3} \\
0 & 0 \\
& h^{2} w_{3} & 0 \\
h^{2} w_{3}-H_{2} & 0 \\
* & -w_{3}
\end{array}\right]<0 \text { (31) }
$$

$\left[\begin{array}{ll}w_{1} & w_{2} \\ w_{2}^{T} & w_{3}\end{array}\right]>0$

with:

$$
\begin{aligned}
& \Gamma_{11}=2 \alpha P+P A+A^{T} P+h^{2} w_{1}+h^{2} A^{T} w_{2}^{T}+h^{2} w_{2} A+Q_{1} \\
& \quad-e^{-2 \alpha h} w_{3}+\gamma^{2} H_{1} \\
& \Gamma_{12}=P A_{h}+h^{2} w_{2} A_{h}+e^{-2 \alpha h} w_{3} \\
& \Gamma_{22}=-(1-d) e^{-2 \alpha h} Q_{1}-e^{-2 \alpha h} w_{3}+\beta^{2} H_{2}
\end{aligned}
$$

\section{Proof of Corollary 1}

Choose a Lyapunov-Krasovskii functional as:

$$
V(t, x(t))=V_{1}(t, x(t))+V_{2}(t, x(t))+V_{3}(t, x(t))
$$

where $V_{1}, V_{2}, V_{3}$ are defined in equation (14), and in order to remove the term $\dot{x}(t-h(t))$, the matrices $Q_{2}$ and $Q_{3}$ are set to be zero. The proof is completed.

In practice, the systems almost present some uncertainties because it is very difficult to obtain an exact mathematical model due to environmental noise, uncertain or slowly varying parameters, etc. Therefore, it will be interesting to study the case of a neutral system which presents nonlinear perturbations and time varying uncertain matrices with appropriate dimensions subject to the norm-bounded forms. This system is shown in the following section.

\section{Robust Exponential Stability for Neutral Time Varying Delay Systems with Nonlinear Perturbations and Norm- bounded Uncertainties}

The robust stability for the perturbed and uncertain systems with time varying delays presents an important focus that described systems more physical than other ones.

Thus, this section handles the case that system (1) is with norm-bounded uncertainties. The nonlinear uncertain neutral delay system (1) is now described by the following state equation:

$$
\begin{aligned}
\dot{x}(t) & =(A+\Delta A(t)) x(t)+\left(A_{h}+\Delta A_{h}(t)\right) x(t-h(t)) \\
& +C \dot{x}(t-h(t))+f(t, x(t))+g(t, x(t-h(t))) \\
x(t) & =\phi(t), \quad \dot{x}(t)=\varphi(t), \quad \forall t \in[-h, 0]
\end{aligned}
$$

where $\Delta A(t)$ and $\Delta A_{h}(t)$ denote the parameter uncertainties vector described by:

$\left[\Delta A(t) \quad \Delta A_{h}(t)\right]=D F(t)\left[\begin{array}{ll}E & E_{h}\end{array}\right]$

where $D, E, E_{h}$ are constant real matrices with appropriate dimensions, and $F(t) \in \mathfrak{R}^{n \times m}$ is a time varying matrix function satisfying $F^{T}(t) F(t) \leq I, \forall t>0$.

\section{Theorem 2}

For given scalars $\alpha>0$ and $h>0$, the uncertain nonlinear neutral system described by (33) is robustly exponentially stable with a decay rate $\alpha$ if there exist positive definite 
symmetric $n \times n$ matrices $P, w_{1}, w_{3}, Q_{1}, Q_{3}$, $H_{1}, H_{2}$, any $n \times n$ matrices $w_{2}, Q_{2}$ and positive scalar $\varepsilon$ such that the following LMIs hold:

$$
\begin{aligned}
& \Upsilon=\left[\begin{array}{cccccc}
\Upsilon_{11} & \Upsilon_{12} & \Upsilon_{13} & -e^{-2 \alpha h} w_{2}^{T} & \Upsilon_{15} & \Upsilon_{16} \\
* & \Upsilon_{22} & \Upsilon_{23} & e^{-2 \alpha h} w_{2}^{T} & \Upsilon_{25} & \Upsilon_{26} \\
* & * & \Upsilon_{33} & 0 & \Upsilon_{35} & \Upsilon_{36} \\
* & * & * & -e^{-2 \alpha h} w_{1} & 0 & 0 \\
* & * & * & * & \Upsilon_{55} & \Upsilon_{56} \\
* & * & * & * & * & \Upsilon_{66} \\
* & * & * & * & * & * \\
* & * & * & * & * & * \\
* & * & * & * & * & * \\
* & * & * & * & * & *
\end{array}\right. \\
& \left.\begin{array}{cccc}
h A^{T} w_{3} & A^{T} Q_{3} & P D+h^{2} w_{2} D+Q_{2} D & \varepsilon E^{T} \\
h A_{h}^{T} w_{3} & A_{h}^{T} Q_{3} & 0 & \varepsilon E_{h}^{T} \\
h C^{T} w_{3} & C^{T} Q_{3} & 0 & 0 \\
0 & 0 & 0 & 0 \\
0 & 0 & h^{2} w_{3} D+Q_{3} D & 0 \\
0 & 0 & h^{2} w_{3} D+Q_{3} D & 0 \\
-w_{3} & 0 & h w_{3} D & 0 \\
* & -Q_{3} & Q_{3} D & 0 \\
* & * & -\varepsilon I & 0 \\
* & * & * & -\varepsilon I
\end{array}\right]<0
\end{aligned}
$$$$
\left[\begin{array}{cc}
w_{1} & w_{2} \\
w_{2}^{T} & w_{3}
\end{array}\right]>0,\left[\begin{array}{ll}
Q_{1} & Q_{2} \\
Q_{2}^{T} & Q_{3}
\end{array}\right]>0
$$

where $\Upsilon_{i j}=\Sigma_{i j}, i, j=1, \ldots, 6$, are the same as defined in the Theorem 1.

\section{Proof of Theorem 2}

The proof follows from the proof of Theorem 1. Let us reconsider the matrix inequality $\Sigma<0$ defined in theorem 1. We shall replace $A$ and $A_{h}$ with $A(t)=A+D F(t) E$ and $A_{h}(t)=A+D F(t) E_{h}$, respectively in $\Sigma<0$ and rewrite the resulting inequality as:

$$
\Sigma+H F(t) G+G^{T} F^{T}(t) H^{T}<0
$$

$H^{T}=D^{T}\left[P+h^{2} w_{2}^{T}+Q_{2}^{T} 000 h^{2} w_{3}+Q_{3} h^{2} w_{3}+Q_{3} h^{2} w_{3} Q_{3}\right]$ $G=\left[\begin{array}{llllllll}E & E_{h} & 0 & 0 & 0 & 0 & 0 & 0\end{array}\right]$

It follows from Lemma 4, that there exist a scalar $\varepsilon$ such that the inequality (37) is equivalent to:

$\Sigma+H F(t) G+G^{T} F^{T}(t) H^{T} \leq \Sigma+\varepsilon^{-1} H H^{T}+\varepsilon G^{T} G=\Omega(38)$
Applying Schur complements, we obtain the linear inequality given in (35). Thus, $\Omega<0$ is equivalent to $\Upsilon<0$ which ensures system (33) is robustly exponentially stable in the sense of the argument given in the proof of Theorem 1.This completes the proof.

\section{Remark 3}

The main results developed in this paper are delay dependent exponential stability conditions for perturbed systems (1), (30) and (33). The main improvement is that the proposed Theorems 1 and 2 and Corollary 1 are obtained without using model transformation techniques, bounding techniques for cross terms and free weighting matrices. Hence, it will be shown in the following section that the obtained results are less conservative than those in the literature.

\section{Illustrative Examples}

In this section, numerical examples are illustrated in order to demonstrate the application of the theorems given in this paper in order to show the effectiveness of our new stability criteria via making some comparison analysis with the existing results from the literature.

\section{Example 1}

Consider the nonlinear neutral system (1) with the following system matrices:

$$
\begin{aligned}
& A=\left[\begin{array}{cc}
-2 & 0 \\
0 & -0.9
\end{array}\right], A_{h}=\left[\begin{array}{cc}
-1 & 0 \\
-1 & -1
\end{array}\right], C=\left[\begin{array}{cc}
0.1 & 0 \\
0 & 0.1
\end{array}\right], \\
& \gamma=0.05, \beta=0.1
\end{aligned}
$$

By applying Theorem 1 to the above system, one can obtain the maximum upper bounds of the time delay $h$ under different values of $d$ and $\alpha$ as shown in Table 1 .

Clearly, one can see from the Table 1 that the proposed method provides less conservative results than previous method investigated in [8] and [11].

\section{Example 2}

Consider the nonlinear time delay system (30) with: 
$A=\left[\begin{array}{cc}-1.2 & 0.1 \\ -0.1 & -1\end{array}\right], A_{h}=\left[\begin{array}{cc}-0.6 & 0.7 \\ -1 & -0.8\end{array}\right], \alpha=0, \gamma \geq 0, \beta \geq 0$

Applying the methods in $[6,8,11,13,19,26$, 27] and Corollary 1, the maximum values of $h$ for the asymptotic stability of system under consideration is listed in the following Table 2. It is easy to see that the stability criterion in this paper gives less conservative result than those obtained in $[8,11,19]$. If $d<1$, the maximum allowable delay $h$ obtained is the same as in [19]. When $d \geq 1$, the method [19] becomes infeasible; however our approach has succeeded to give a better result than [8] and [11] which present also an improved result than those developed in $[6$, $16,23,27]$.

\section{Example 3}

Time delay and nonlinear perturbations (33) with: $A=\left[\begin{array}{cc}-0.9 & 0.2 \\ 0.1 & -0.9\end{array}\right], A_{h}=\left[\begin{array}{cc}-1.1 & -0.2 \\ -0.1 & -1.1\end{array}\right], C=\left[\begin{array}{cc}-0.2 & 0 \\ 0.2 & -0.1\end{array}\right]$ $\gamma=0.05, \beta=0.1\left[\Delta A(t) \Delta A_{h}(t)\right]=D F(t)\left[\begin{array}{ll}E & E_{h}\end{array}\right]$, $F^{T}(t) F(t) \leq I, \quad D=0.05^{*} I, \quad E=E_{h}=I$
Table 3 gives out the maximal allowable delay $h$ for the robust exponential stability of system (33) by application of the new Theorem 2.

\section{Conclusion}

In this paper, a new delay dependent robust exponential stability criterion for neutral systems with time varying delays and nonlinear perturbations is established. By deriving the stability criterion in terms of LMI and using of Lyapunov-krasovskii parameter dependent functional, the upper bound of the time delays can be calculated easily. The main improvement from the proposed method is that the results have been obtained without using model transformation techniques and bounding techniques for cross terms, and without introducing free weighting matrices. Numerical examples have clearly shown that the criteria derived herein are considerably less conservative than those in the literature.

Table 1. Upper bounds of time delays in Example 1

\begin{tabular}{llccc} 
& $\alpha$ & 0.1 & 0.3 & 0.5 \\
\hline \multirow{3}{*}{$h(d=0)$} & {$[11]$} & 1.2987 & 0.8651 & 0.6823 \\
& {$[8]$} & 1.2999 & 0.8781 & 0.6917 \\
& Theorem 1. & 1.4856 & 0.9413 & 0.7179 \\
\hline \multirow{3}{*}{$h(d=0.5)$} & {$[11]$} & 0.9386 & 0.7135 & 0.6091 \\
& {$[8]$} & 0.9442 & 0.7275 & 0.6096 \\
& Theorem 1. & 1.3330 & 0.8094 & 0.6503 \\
\hline \multirow{3}{*}{$h(d=0.9)$} & {$[11]$} & 0.5326 & 0.5124 & 0.4725 \\
& {$[8]$} & 0.5471 & 0.5015 & 0.4650 \\
& Theorem 1. & 0.7256 & 0.5752 & 0.4812 \\
\hline
\end{tabular}

Table 2. Upper bounds of time delays in Example 2 for different conditions

\begin{tabular}{|c|c|c|c|c|c|c|}
\hline & \multicolumn{3}{|c|}{$\gamma=0, \beta=0.1$} & \multicolumn{3}{|c|}{$\gamma=0.1, \beta=0.1$} \\
\hline & $d=0$ & $d=0.5$ & $d \geq 1$ & $d=0$ & $d=0.5$ & $d \geq 1$ \\
\hline [19] & 2.7758 & 1.1461 & & 1.8959 & 1.0143 & \\
\hline [8] & 2.7423 & 1.1425 & 0.7355 & 1.8753 & 1.0097 & 0.7147 \\
\hline [11] & 2.7433 & 1.1439 & 0.7355 & 1.8762 & 1.0108 & 0.7147 \\
\hline Corollary 1. & 2.7758 & 1.1461 & 0.7368 & 1.8959 & 1.0143 & 0.7163 \\
\hline
\end{tabular}

Table 3. Upper bounds of time delays in Example 3 for different conditions

\begin{tabular}{|c|c|c|c|c|c|c|}
\hline$\alpha$ & 0 & 0.1 & 0.3 & 0.5 & 0.7 & 0.9 \\
\hline$h(d=0)$ & 1.3838 & 1.0550 & 0.7668 & 0.6199 & 0.5252 & 0.4528 \\
\hline$h(d=0.5)$ & 1.0126 & 0.8457 & 0.6642 & 0.5599 & 0.4852 & 0.4199 \\
\hline$h(d=0.9)$ & 0.5304 & 0.4662 & 0.3763 & 0.3134 & 0.2634 & 0.2145 \\
\hline
\end{tabular}




\section{REFERENCES}

1. AMRI, I., D. SOUDANI, Robust Exponential Stability of Uncertain Perturbed Systems with Time Varying Delays, accepted in IFAC-12th LSS symposium: Theory and Applications, France, 2010.

2. AMRI, I., D. SOUDANI, M. BENREJEB, New Condition for Exponential Stability of Linear Time Delay Systems: LMI Approach, 9th International Conference on Sciences and Techniques of Automatic Control \& Computer Engineering STA, Sousse, Tunisia, 2008.

3. AMRI, I., D. SOUDANI, M. BENREJEB, Exponential Stability and Stabilization of Linear Systems with Time Varying Delays, 6th International Multi-Conference on Systems, Signals and Devices SSD, Djerba Tunisia, 2009.

4. AMRI, I., D. SOUDANI, M. BENREJEB, Delay Dependent Robust Exponential Stability for Uncertain Systems with Time Varying Delays and Nonlinear Perturbations, 10th International Conference on Sciences and Techniques of Automatic Control \& Computer Engineering STA, Hammamet Tunisia, 2009.

5. BOYD, S., L. EL GHAOUI, E. FERON, V. BALAKRISHNAN, Linear Matrix Inequalities in Systems and Control Theory, SIAM, Philadelphia, 1994.

6. CAO, Y.-Y., J. LAM, Computation of Robust Stability Bounds for Time Delay Systems with Nonlinear Time Varying Perturbation, International Journal of Systems Science, Vol. 31, 2000, pp. 359-365.

7. CAO, D. Q., P. HE, K. ZHANG, Exponential Stability Criteria of Uncertain Systems with Multiple Time Delays, Journal of Mathematical Analysis and Applications, Vol. 283, 2003, pp. 362-374.

8. CHEN, Y., A. XUE, R. LU, S. ZHOU, On Robustly Exponential Stability of Uncertain Neutral Systems with Time Varying Delays and Nonlinear
Perturbations, Nonlinear Analysis 68, 2008, pp. 2464-2470.

9. CHEN, W. H., Z. H. GUAN, X. LU, Delay Dependent Exponential Stability of Uncertain Stochastic Systems with Multiple Delays: an LMI Approach, Systems \& Control Letters, Vol. 54, 2005, pp. 547-555.

10. DE SOUZA, C. E., X. LI, Delaydependent Robust $H \infty$ Control of Uncertain Linear State-delayed Systems, Automatica, Vol. 35, 1999, pp.1313-1321.

11. FANG, Y., Z. XU, N. CHEN, Robust Exponential Stability of Uncertain Neutral Systems with Time Delays and Nonlinear Perturbations, Proceedings of the 7th World Congress on Intelligent Control and Automation, China, 2008.

12. GU, K., V. L. KHARITONOV, J. CHEN, Stability of Time-delay Systems, Boston: Birkhauser, 2003.

13. HAN, Q.-L., On Robust Stability of Linear Neutral Systems with Nonlinear Parameter Perturbations, Proceeding of the American Control Conference, Boston Massachusetts, 2004, pp. 2027-2032.

14. HAN, Q.-L., On Robust Stability of Neutral Systems with Time Varying Discrete Delay and Norm-bounded Uncertainty, Automatica, Vol. 40, 2004, pp. 1087-1092.

15. KACEM, W., M. CHABAANE, D. MEHDI, M. KAMOUN, Delay Dependent Robust Exponential Stability and Stabilization of Uncertain Statedelayed Systems, Studied in Informatics and Control, Vol. 15, Nr. 1, 2006.

16. KHARITONOV, V. L., S. MONDIÉ, J. COLLADO, Exponential Estimates for Neutral Time-delay Systems, IEEE Transactions on Automatic Control, Vol. 50, Nr. 5, 2005, pp. 666-670.

17. KOLMANOVSKII, V., V. MYSHKIS, Applied Theory of Functional Differential Equations, Kluwer Academic Publishers, Boston, 1992.

18. KUANG, Y., Delay Differential Equations with Application in Population Dynamics, Mathematics in 
Science and Engineering, Vol. 191, Academic Press, San Diego, 1993.

19. KWON, O. M., J. H. PARK, S. M. LEE, On Robust Stability Criterion for Dynamic Systems with Time-varying Delays and Nonlinear Perturbations, Applied Mathematics and Computation, Vol. 203, 2008, pp. 937-942.

20. KWON, O. M., J. H. PARK, Exponential Stability for Time Delay Systems with Interval Time-Varying Delays and Nonlinear Perturbations, Journal of Optimization Theory and Applications, Springer, Vol. 139, 2008, pp. 277-293.

21. KWON, O. M., J. H. PARK, Matrix Inequality Approach to Novel Stability Criterion for Time Delay Systems with Nonlinear Uncertainties, Journal of Optimization Theory and Applications, Vol. 126, 2005, pp. 643-656.

22. MOEZ BELHAOUANE, M., R. MTAR, H. BELKHIRIA AYADI, N. BENHADJ BRAIEK, An LMI Technique for the Global Stabilization of Nonlinear Polynomial Systems, International Journal of Computers, Communications \& Control, Vol. 4, Nr. 4, 2009, pp. 348-348.
23. PHAN, T. N., N. P. VU, Robust Exponential Stability and Stabilization of Linear Uncertain Polytopic Time Delay Systems, Journal Control Theory Application, Vol. 6, Nr. 2, 2008, pp. 163-170.

24. SAADAOUI, K., S. ELMADSSIA, M. BENREJEB, Stabilizing First-order Controllers for n-th Order All Pole Plants with Time Delay, 16th Mediterranean Conference on Control and Automation, France, 2008.

25. YAKUBOVICH, V. A., S-procedure in Nonlinear Control Theory, Ser. Matematika: Vestnik Leningradskogo Universiteta, 1971, pp. 62-77.

26. ZHANG, J., P. SHI, J. QIU, Robust Stability Criteria for Uncertain Neutral System with Time Delay and Nonlinear Uncertainties, Chaos, Solitons \& Fractals, Vol. 38, 2008, pp. 160-167.

27. ZOU, Z., Y. WANG, New Stability Criterion for a Class of Linear Systems with Time Varying Delay and Nonlinear Perturbations, IEE Proceedings Control Theory and Applications, Vol. 153, Nr. 5, 2006, pp. 623-626. 